\title{
Genome
}

\section{Transposable element landscapes illuminate past evolutionary events in the endangered fern Vandenboschia speciosa}

\begin{tabular}{|r|l|}
\hline Journal: & Genome \\
\hline Manuscript ID & gen-2021-0022.R1 \\
\hline Danuscript Type: & Article \\
\hline Author: & 13 -Sep-2021 \\
\hline Komplete List of Authors: & $\begin{array}{l}\text { Ruiz-Ruano, Francisco; UNIVERSIDAD DE GRANADA } \\
\text { Navarro-Domínguez, Beatriz; UNIVERSIDAD DE GRANADA } \\
\text { Camacho, J P; Universidad de Granada } \\
\text { Garrido-Ramos, Manuel; UNIVERSIDAD DE GRANADA }\end{array}$ \\
\hline $\begin{array}{r}\text { Is the invited manuscript for } \\
\text { consideration in a Special } \\
\text { Issue? : }\end{array}$ & Not applicable (regular submission) \\
\hline &
\end{tabular}

\section{SCHOLARONE ${ }^{m}$ Manuscripts}


Transposable element landscapes illuminate past evolutionary events in the endangered fern Vandenboschia speciosa

\section{Francisco J. Ruiz-Ruano ${ }^{1,2,3}$, Beatriz Navarro-Domínguez ${ }^{1,4}$, Juan Pedro M. Camacho ${ }^{1}$ and} Manuel A. Garrido-Ramos ${ }^{1, *}$

${ }^{1}$ Departamento de Genética, Facultad de Ciencias, Universidad de Granada, Granada, Spain ${ }^{2}$ Department of Organismal Biology, Systematic Biology, Evolutionary Biology Centre, Uppsala University, Uppsala, Sweden

${ }^{3}$ School of Biological Sciences, University of East Anglia, Norwich, United Kingdom

${ }^{4}$ Department of Biology, University of Rochester, Rochester, New York 14627

*Correspondence: mgarrido@ugr.es 
41 Abstract

Vandenboschia speciosa is an endangered tetraploid fern species with a large genome (10.5 Gb). Its geographical distribution is characterized by disjoined tertiary flora refuges, with relict populations that survived past climate crises. Here we analyze the transposable elements (TEs) and found that they comprise about $76 \%$ of the $V$. speciosa genome, thus being the most abundant kind of DNA sequences in this gigantic genome. $V$. speciosa genome is composed of $51 \%$ and $5.6 \%$ of Class I and Class II elements, respectively. LTR retrotransposons were the most abundant TEs in this species (at least $42 \%$ of the genome), followed by non-LTR retrotransposons that constituted at least $8.7 \%$ of the genome of this species. We introduce an additional analysis to identify the nature of non-annotated elements (19\% of the genome). A BLAST search of the non-annotated contigs against the $V$. speciosa TE database allowed determining the identity of almost half of them, which were most likely diverged sequence variants of the annotated TEs. In general, TE composition in $V$. speciosa resembles TE composition in seed plants. In addition, repeat landscapes revealed three episodes of amplification for all TEs, most likely due to demographic changes associated to past climate crises.

Keywords: climate crisis, demographic changes, endangered species, ferns, genome size, relict populations, tetraploidy, transposable elements, Vandenboschia speciosa.

60 


\section{Introduction}

Transposable elements (TEs) are ubiquitous components of eukaryotic genomes that are considered drivers of genome evolution (Böhne et al. 2008; Belyayev 2014; Bourque et al. 2018), with relevant impact on both genome regulation (Slotkin and Martienssen 2007; Feschotte 2008; García-Pérez et al. 2016) and size (Gregory 2005; Bennetzen and Park 2018). A comprehensive analysis of how TE landscape contributes to a particular genome is thus relevant from a structural, functional and evolutionary perspective. Next-Generation Sequencing (NGS) and high-throughput in silico analysis of NGS reads have transformed the study of repetitive DNA, especially since the introduction of the RepeatExplorer (RE) pipeline which allows the identification and characterization of thousands of repetitive DNA elements on short NGS reads, by employing graph-based clustering of sequence reads (Novák et al. 2010, 2013, 2020b). Furthermore, the efficiency of repetitive DNA mining can be increased by means of the recursive application of the RE clustering algorithm combined with filtering out, at each round, the reads containing already known repetitive families (Ruiz-Ruano et al. 2016). Generated contigs are then properly annotated with appropriate software such as DANTE (http://repeatexplorer.org/), which tracks the REXdb database (Neumann et al. 2019).

As a general rule, there is a relationship between TE abundance and genome size, which contributes to explain the C-value paradox (Gregory 2005; Bennetzen and Park 2018). Indeed, it has been recently proved that, in land plants, genome size increases proportionally to repetitive DNA amount, reaching up to proportions of around $80 \%$ of repetitive DNA in large genomes (Novák et al. 2020a). Curiously, this trend is shifted in genomes larger than $10 \mathrm{~Gb}$ and the largest genomes might have about $55 \%$ of repetitive DNA, probably by the slow degradation of repeats over time (Novák et al. 2020a). Notwithstanding this, TE accumulation is not the only cause for genome size increase in plants, as polyploidization is considered to play a major role in plant genome size evolution (Alix et al. 2017; Vicient and Casacuberta 2017). In fact, it has been suggested that polyploidization might be the major factor contributing to the high chromosome numbers and large genomes in ferns (Klekowski and Baker 1966; Klekowski 1972; Wagner and Wagner 1980; Nakazato et al. 2008; Dyer et al. 2013; Marchant et al. 2019).

In this context, biological, life-history and genomic features, together with the phylogenetic position within vascular plants, make Vandenboschia speciosa an attractive species for a genome-wide analysis with the aim to contribute to the knowledge of the impact of TEs in genome size and evolution in ferns. $V$. speciosa is a tetraploid fern species with a huge genome (10.5 Gb) (Manton 1950; Manton et al. 1986; Obermayer et al. 2002; Ebihara et al. 2007). This species is an endangered fern whose habitat is currently threatened by destruction and over- 
harvesting (Rumsey et al. 1999). It is a rare European-Macaronesian endemism, the only representative of a genus which has a primarily tropical distribution, with a current geographical distribution characterized by disjointed tertiary flora refuges in the European Atlantic coast and the Macaronesian islands (Canaries, Madeira and Azores), composed of relic populations with very few individuals that survived past climate crises (Rumsey et al. 1999). We have found that most DNA sequences in the genome of the fern Vandenboschia speciosa are TEs and that its specific TEs composition is similar to seed plants TEs composition. In addition, we analyzed repeat landscapes to investigate possible amplification events for each TE, in order to get insights on recent evolutionary pathways of these elements that could be important to understand the present relict distribution of this endangered species.

\section{Materials and Methods}

\section{Materials}

Vandenboschia speciosa sporophytes were collected at one out of seven populations located in the Alcornocales Natural Park (Cádiz, Spain): Canuto de Ojén-Quesada (OJEN). Sporophytes were frozen in liquid nitrogen in the field and stored at $-80^{\circ} \mathrm{C}$. Genomic DNA (gDNA) was isolated using the DNeasy plant Mini kit (Quiagen). Pools of DNAs were generated from sets of five specimen DNAs and sequenced by Illumina HiSeq-2000 PE 2x101 nt technology, yielding about $16 \mathrm{~Gb}$ data ( $1.5 \mathrm{x}$ coverage). Illumina sequencing data can be accessed at Sequence Read Archive (SRA) database under the BioProject PRJNA387541.

TE assembly and annotation

We performed an in-depth assembly of repetitive elements using RE (Novák et al. 2010, 2013, 2020b). For this, we first performed a quality trimming with Trimomatic (Bolger et al. 2014) to keep read pairs without adapters and a minimum quality of Q20. Then we randomly selected 2 x 2,000,000 Illumina reads with SeqTK (https://github.com/lh3/seqtk) to run RE with default options. After one RE run, we extracted the most representative contigs for every cluster, specifically those representing up to a half of total cluster coverage with a custom script (https://github.com/fjruizruano/satminer/blob/master/rexp_get_contigs.py) and filtered out the reads from the original library that matches them using DeconSeq (Schmieder and Edwards 2011). Then, we randomly selected a new set of $2 \times 2,000,000$ reads from the filtered libraries, that were clustered with RE in a second round. Performing additional rounds of clustering and 
144 filtering had shown to be highly successful for satellite DNA (Ruiz-Ruano et al. 2016), as it allows

145 detecting repetitive elements which, due to their low abundance, had gone unnoticed because 146 their signals were masked by those of highly abundant elements. We annotated the resulting 147 contigs by the DANTE software (http://repeatexplorer.org/) with the iterative search option and using as a reference the Viridiplantae v3.0 of REXdb (Neumann et al. 2019), i.e. a curated database for protein domains of plant repetitive. We considered separately the most conservative annotation in the "Final Classification" field. This classification is based on multiple top hits (the best hit + all other hits with score $>=80 \%$ of the score of the best hit). But sequences are classified on the deepest level showing no conflict among hits (Neumann et al. 2019). Thus, for example, a conflict between Class_I|LTR|Ty3/gypsy|chromovirus|Reina and

Class_I|LTR|Ty3/gypsy|chromovirus (Neumann et al. 2019). We annotated the contigs from the two RE rounds, excluding the "Simple_repeat" and "Low_complexity" contigs, and labeling the non-annotated contigs as "Unknown". Then, we used the msatcommander software (Faircloth 2008) to search for perfect microsatellite arrays (from 1 to $6 \mathrm{nt}$ of monomer size) and removed arrays with 20 or more nucleotides. This is the minimum sensibility that RepeatMasker has to detect a microsatellite array. In addition, we screened the database with the CD-HIT program (Fu et al. 2012) using the options "-M 0 -aS 0.8 -c 0.8 -G 0 -g 1" in order to detect redundant contigs with at least a $80 \%$ of identity showing discrepant annotations. We did not find such kind of discrepancies in this sanity check. Finally, we combined all annotated RE contigs in a single database. As we were focused here on the study of TEs, we removed other repetitive elements from the final database, such as satDNA (Ruiz-Ruano et al. 2019a) and plastome sequences (RuizRuano et al. 2019b), which had previously been characterized in V. speciosa. In addition, we assembled the $45 \mathrm{~S}$ and $5 \mathrm{~S}$ ribosomal DNAs with the MITObim software (Hahn et al. 2013), using Tetraplodon fuegianus 45S (GenBank accession number KU095852) and Marsilea quadrifolia 5S (GenBank accession number FR694363) as seeds. We then annotated the three types of elements with RepeatMasker (Smit et al. 2015) with "nolow" and "no_is" options and removed the contigs matching with these non-TE repetitive elements. Finally, we included the DANTE annotation for each RE cluster to the contigs IDs in the FASTA file with the RepeatMasker's format using a custom script (https://github.com/fjruizruano/ngsprotocols/blob/master/rexp_annot.py). The resulting database was deposited in FigShare (https://figshare.com/articles/dataset/Supplementary_Dataset_for_The_repeatome_of_the_e ndangered_fern_Vandenboschia_speciosa_/12124503). 
In order to estimate abundance and divergence for each annotated element, we aligned 5 million of randomly selected read pairs to the consensus sequences in the resulting RE database, using RepeatMasker

with

a

custom

script (https://github.com/fjruizruano/satminer/blob/master/repeat_masker_run_big.py). We used the calcDivergenceFromAlign.pl built-in tool of RepeatMasker to obtain a histogram of the Kimura 2-Parameter divergence for each element. Next, we transformed the abundance values to express them as genome proportion by dividing the number of aligned nucleotides by the total number of nucleotides in the selection of 10 million read pairs. The resulting histograms (hereafter referred to as Repeat Landscapes, RLs) were plotted in R.

\section{Results}

192

A first run of RE analysis allowed identifying 495 clusters of repetitive DNA sequences. However, an additional run of filtering+RE increased this figure up to 1,271 which were subsequently annotated by DANTE as TEs (Table 1). According to these annotations, TEs comprise at least $76 \%$ of the $V$. speciosa genome (Table 1 ).

As Table 1 shows, the $V$. speciosa genome has almost ten times the amount of retrotransposons related sequences (Class I elements) than DNA transposons related sequences (Class II elements), both kinds representing about $51 \%$ and $5.6 \%$ of the genome, respectively. By far, the most abundant sequences in $V$. speciosa are LTR retrotransposons ( $81.9 \%$ of Class I elements), belonging to two superfamilies (Ty1/Copia and Ty3/Gypsy), each representing at least about $18.5 \%$ of the genome (Table 1). Only a percentage of Ty1/Copia (47\% of Copia elements), could be assigned to a particular family, predominating Ale and Tork (Table 1). On the contrary, most Ty3/Gypsy sequences could be further annotated (99.3\%), predominating the OTA group with Athila as the most representative element among Ty3/Gypsy elements (6.7\% of the genome). LINE (Non-LTR retrotransposons TEs) sequences represent unusual amount in $V$. speciosa genome (at least $8.7 \%$ of the genome). Among DNA transposons, the higher amount of sequences belonged to EnSpm-CACTA elements, as it represents $62 \%$ of identified transposon sequences, followed by Sola1 (36\%) and Helitron (1.6\%).

Almost $57 \%$ of the genome was annotated, thus remaining, at first instance, about $19 \%$ of the genome composed of "Unknown" elements (Table 1). In an effort to further characterize the non-annotated contigs, we blasted their sequences to the generated $V$. speciosa TE database, and found an important set of contigs that showed homology to some of the 
annotated sequences. Specifically, almost 32\% of the "Unknown" sequences showed homology with annotated LTR elements (about 7\% LTR/Copia and about 23\% LTR/Gypsy), 4.6\% with annotated LINEs and about $8 \%$ with DNA transposons (Table 2). Therefore, about $44 \%$ of nonannotated sequences could be somewhat identified by this procedure (Table 2 ). This allowed identifying about 8.5\% (6.1\% LTR, 0.9 LINE and 1.5 DNA transposons) of the $V$. speciosa genome as divergent variant sequences of TEs already annotated in Table 1. This raised the frequency of identified TEs till $65.4 \%$, whereas the remaining $10.7 \%$ of TEs in the genome might be highly divergent or fern-specific TE sequences (Table 2).

It was remarkable to find that all TE superfamilies found within the genome of $V$. speciosa showed a similar profile for the Repeat Landscapes (RLs) built by comparing abundance and divergence of sequence variants (Figure 1). Thus, the landscapes are characterized by the presence of two to three well-defined peaks of abundance in most of the elements: one more diffuse representing sequences placed around 18\% divergence, one peak around 13\% divergence and the most conspicuous peak being around $4 \%$ of sequence divergence. It was also clear that this latter peak showed some slight differences among elements, as it was placed about 5\% for LTR/Copia-Ale and LTR/Copia-Tork, 3\% for DNA/Sola1 and LTR/Copia-Gymcoll, 2\% for LINE and Penelope as well as for LTR/Gypsy|chromovirus (see Figure 1).

\section{Discussion}

TEs largely contribute to $V$. speciosa genome size

To date, contrasting to other vascular plants, only the genomes of two heterosporous and one homosporous ferns have been sequenced (Sessa et al. 2014; Li et al. 2018; Marchant et al. 2019), and some other fragmentary data on TEs from a few fern genomes are available (Dyer et al. 2013; Wolf et al. 2015). However, because of their phylogenetic position, ferns are crucial for investigating TEs as well as other genomic traits. We wanted to contribute to this knowledge taking advantage of the development of new robust tools for the analysis of NGS reads. In this context, our present results revealed that $76 \%$ of the $V$. speciosa genome is composed of TEs, considerably improving our previous quantitative estimates of TEs in $V$. speciosa obtained after a single RE run (Ruiz-Ruano et al. 2019a). This is the highest proportion of TEs hitherto found in a fern genome (Wolf et al. 2015; Li et al. 2018; Marchant et al. 2019). Furthermore, our research confirm that TEs are the major component of the repeatome of $V$. speciosa while its tandem repetitive component comprised by satellite DNAs (about $0.4 \%$ of the genome) and 
microsatellites (about $2 \%$ of the genome) does not explain the huge genome size in this species (Ruiz-Ruano et al. 2019a).

The large fern genomes, especially homosporous ferns (average genome size $12 \mathrm{~Gb}$; Sessa and Der 2016), are proposed to be paleopolyploid (reviewed in Barker 2013) behaving as diploid, and are characterized by extremely high numbers of chromosomes. As a result, polyploidization has been suggested as the major factor contributing to the high chromosome numbers and large genomes in ferns (Klekowski and Baker 1966; Klekowski 1972; Wagner and Wagner 1980; Nakazato et al. 2008; Dyer et al. 2013; Marchant et al. 2019). V. speciosa is considered to be a tetraploid species (Manton 1950; Manton et al. 1986; Obermayer et al. 2002), probably an allotetraploid (Ebihara et al. 2007), with 2n=144 chromosomes (Obermayer et al. 2002), which partly explains its large genome $(1 \mathrm{C}=10.52 \mathrm{~Gb})$. However, we show here that TEs might be the main cause of genome size increase in this species, as they constitute $3 / 4$ of genome sequences. In fact, recent papers claim that differences in fern genome size are attributable to TEs, and that fern repeat proportions are comparable to those of flowering plants (Li et al. 2018; Marchant et al. 2019). After analyzing genome size and spore size variation in the Asplenium monanthes fern complex, Dyer et al. (2013) concluded that other mechanisms, in addition to polyploidy, should explain genome size variation in ferns, and suggested "retrotransposon driven changes" as a possible cause. Our present results give support to this inference as retrotransposons actually constitute the immense majority of TEs in $\mathrm{V}$. speciosa. These data agree with the assumption that both TE transposition and polyploidization are considered major players in genome size evolution of plants (Alix et al. 2017; Vicient and Casacuberta 2017). In fact, Marchant et al. (2019) have recently found, in the model fern Ceratopteris richardii (11.25 Gb; $n=39)$, evidence suggesting that a single ancient polyploidy event and TE expansion both explain the large fern genomes, in resemblance to flowering plants. Furthermore, members of the fern order Salviniales (heterosporous ferns) that have smaller genome sizes than homosporous ferns also show differences in their repetitive content that explains some of the nearly threefold difference in genome size between Salvinia (Salvinia cucullata, $0.26 \mathrm{~Gb} ; 25 \%$ of the genome are TEs) and Azolla (Azolla filiculoides, $0.75 \mathrm{~Gb} ; 50 \%$ of the genome are TEs) (Li et al. 2018), suggesting that TE expansion appears to have been ubiquitous in ferns.

TE composition in V. speciosa resembles TE composition in seed plants

In contrast to animal genomes, LTR retrotransposons are the most abundant TEs in seed plant genomes (Wicker et al. 2007; López-Flores and Garrido-Ramos 2012). Likewise, LTR 
retrotransposons are the most abundant TE sequences in a few fern species analyzed up to date (Wolf et al. 2015; Li et al. 2018; Marchant et al. 2019), and they represent about $42 \%$ of $V$. speciosa genome ( $48 \%$ if we take into account the divergent elements identified by BLAST) (Tables 1 and 2). In order to contribute to a better understanding of fern TEs, we identified some familiar ascription among the Ty1/Copia and Ty3/Gypsy TEs. Half of the LTR/Copia sequences that we found (53\%) were classified as generic Ty1/Copia elements. However, the other $47 \%$ belonged specifically to five families (Ale, Tork, Gymcoll, Gymcolll and GymcolV), which are usually present in seed plants but are absent in non-vascular plants (Bryophyta) and Lycopodiophyta (Neumann et al. 2019). Specifically, Gymco elements (I to IV) are specific of gymnosperms, whereas Ale and Tork are common to gymnosperms and flowering plants (Neumann et al. 2019). We did not detect LTR/Copia-Bryco or LTR/Copia-Lyco elements, which are the only families found in Bryophyta and Lycopodiophyta, respectively. Among Ty3/Gypsy elements, chromoviruses represent the oldest and most widespread lineage of Ty3/Gypsy retrotransposons in seed plants (Novikov 2012; Neumann et al. 2019). In consistency, three of these families (CRM, Reina and Galadriel) were present in the genome of $V$. speciosa, the latter being also found in Lycophyta. Notwithstanding, they represent only about $12 \%$ of LTR/Gypsy elements (Table 1). Among the non-chromoviruses ( $87 \%$ of LTR/Gypsy elements), OTA were the only type found in this genome (Table 1 ). Many of the OTA sequences detected (55.7\%) could not be further annotated. Among the remaining OTA elements, Athila was the most represented LTR/Gypsy element in V. speciosa $(6.7 \%$ of the genome; almost the $9 \%$ of the genome if we consider the BLAST analysis), followed by Tat, both being typical of vascular plants, also found in lycophytes (Neumann et al. 2019). Remarkably, we did not find Phygy elements, which are specific of Bryophyta, or Selgy elements which are specific of Lycopodiophyta (Neumann et al. 2019). These results suggest that ferns share more classes of LTR elements with seed plants than with other basal groups of plant phylogeny, whether vascular (Lycophyta) or non-vascular (Bryophyta), in concordance with current phylogenies of vascular plants (Pryer et al. 2001; Schneider et al. 2004; Smith et al. 2006).

LINE retrotransposons comprised about $8.7 \%$ of the $V$. speciosa genome $(9.6 \%$ considering the BLAST results), a very high figure compared with other plant genomes (average < 1\%) (Hřibová et al. 2010; Novikov et al. 2012; Makałowski et al. 2019). This finding was previously pointed out by Wolf et al. (2015), although they estimated lower values (average= $2.2 \%$ ) in the fern species analyzed. Interestingly, Penelope represents $0.54 \%$ of the $V$. speciosa genome, whereas it is rarely identified in plant genomes despite its wide distribution among eukaryotes, including the spike moss Selaginella moellendor (Arkhipova 2006; Novikov et al. 2012; Tollis and Boissinot 2012). 
Finally, DNA transposons constitute about $1 \%-15 \%$ of plant genomes (Novikov et al. 2012; Weiss-Schneeweiss et al. 2015), and their proportion in the genome of V. speciosa (5.67.1\%) was within this range, with CACTA and Sola1 as predominating elements, as in other fern genomes (Li et al. 2018). Interestingly, about $1.6 \%$ of all annotated DNA transposons in $V$. speciosa belong to the order Helitron, a kind of rolling-circle transposons that have demonstrated a tremendous potential for gene shuffling and duplication in plants (Morgante et al. 2005; Thomas and Pritham 2015).

Taken together, the TE composition found in the genome of the fern $V$. speciosa shows high resemblance with seed plants, especially in the case of LTR retrotransposons.

BLAST search of the V. speciosa database allowed the increase of the proportion of identified TEs

Successful TEs annotation depends on the similarity of the TEs found in the studied genome with those available in TE databases, which currently are biased toward model organisms which, in the present case, are phylogenetically distant species. In addition, the sequence of inactive TEs diverges through mutation and drift. Thus, the particular TE landscape in each species is composed of a number of repeats that rapidly diverge both at the intra- and inter-specific levels and this makes it difficult to properly identifying genome-specific sequence variants for each element (Neumann et al. 2019). Therefore, it is conceivable that most of the $19 \%$ of the genome containing non-annotated TEs in V. speciosa is made up of diverged TE sequences. In this respect, we further characterized the non-annotated contigs obtained with RE using a BLAST search of the $V$. speciosa TE database. Overall, we identified by this procedure the nature of an additional $8.44 \%$ proportion of the genome. All together, DANTE annotation and BLAST identification of the non-annotated sequences revealed that TEs represent about $65.3 \%$ of the $V$. speciosa genome, whereas another $10.7 \%$ of TEs consists of unidentified TEs, which most likely were too divergent to be identified with the methods employed here. Anyway, we cannot rule out that some of these unidentified sequences could correspond to fern-specific (even functional) TEs.

\section{Temporal changes in TE abundance}

RLs showed one prominent and two less pronounced peaks of TE abundance relative to sequence divergence (see Figure 1). These peaks represent conspicuous sets of repeats grouped around specific values of sequence divergence (i.e. $4 \%, 13 \%$ and $18 \%$ ). As sequence divergence is due to mutational changes and these are proportional to time, we infer that these three peaks 
are indicative of temporally different TE expansion waves within the genome of this species. The fact that repeat landscape profiles were highly similar for all TEs, we infer that these expansion waves were associated with demographic changes in the ancestral populations of the two analyzed here. Current localities of $V$. speciosa are small disjoined tertiary flora refuges harboring relic populations that survived the glacial cycles. Several important climatic change events during the last 5 my (such as the Messinian Salinity Crisis, the Pliocene-Pleistocene transition with the establishment of Mediterranean climate and extinction of typical tertiary taxa, and the Pleistocene with interglacial cycles) might have influenced evolutionary pathways in V. speciosa resulting from successive contractions of the area of distribution of the species, population fragmentation and isolation leading to bottlenecks eroding genetic variability through genetic drift (Ben-Menni Schuler 2019). Previous research indicated that reduced effective population size can trigger an increase in TE copy number and genome size (Lynch and Conery 2003; García-Guerreiro 2012; Bourgeois and Boissinot 2019). According to these authors, while most new TE insertions would be eliminated by selection in large populations, drift would predominate over selection in small populations and thus TE abundance could eventually increase. It is thus conceivable that successive bottlenecks in V. speciosa could have boosted the massive TE expansions reported here. Similar increases in TE copy numbers in small populations after bottlenecks have also been found in Arabidopsis lyrata (Lockton et al. 2008; Ross-Ibarra et al. 2008) and Drosophila subobscura (García-Guerreiro et al. 2008) as a consequence of strong effect of stochastic events and a reduced efficiency of purifying selection in those populations (reviewed in Bourgeois and Boissinot 2019). Interestingly, it cannot be ruled out that the environmental stresses associated to the mentioned events might also be important factors associated to TE activation (Capy et al. 2000; Kalendar et al. 2000; García-Guerreiro 2012; Chuong et al. 2017; Bourgeois and Boissinot 2019).

\section{Acknowledgments}

This research has been financed by the Spanish Ministerio de Economía y Competitividad and FEDER founds, grant: CGL2010-14856 (subprograma BOS). FJ Ruiz-Ruano was supported by a Junta de Andalucía fellowship (Spain), a postdoctoral fellowship from Sven och Lilly Lawskis fond (Sweden) and a Marie Skłodowska-Curie Individual Fellowship grant agreement 875732 (EU). The Dirección General de Gestión del Medio Natural y Espacios Protegidos of the Consejería de Medio Ambiente y Ordenación del Territorio de la Junta de Andalucía authorized and facilitates the sampling of the material. We are highly indebted to Carmen Rodríguez Hiraldo and to Jaime Pereña Ortiz who, together the team of Agentes de Medio Ambiente of the Consejería, helped us with the sampling procedure. 


\section{References}

Alix, K., Gérard, P.R., Schwarzacher, T., Heslop-Harrison, J.S. (Pat). 2017. Polyploidy and

391

392

393

394

395

396

397

398

399

400

401

402

403

404

405

406

407

408

409

410

411

412

413

414

415

416

417

418

419 interspecific hybridization: partners for adaptation, speciation and evolution in plants. Ann. Bot., 120:183-194.

Arkhipova, I.R. 2006. Distribution and phylogeny of Penelope-like elements in eukaryotes. Syst. Biol., 55: 875-885.

Barker, M.S. 2013. Karyotype and genome evolution in Pteridophytes. In: Leitch I.J. et al. (eds) Plant Genome Diversity. Volume 2, Physical Structure, Behaviour and Evolution of Plant Genomes. Springer-Verlag, Wien, pp 245-253.

Belyayev, A. 2014. Bursts of transposable elements as an evolutionary driving force. J. Evol. Biol., 27: 2573-2584.

Ben-Menni Schuler, S. 2019. Genetic diversity and phylogeographic pattern of four Tertiary-relict fern species: Vandenboschia speciosa (Willd.) G. Kunkel, Diplazium caudatum (Cav.) Jermy, Pteris incompleta Cav. and Culcita macrocarpa C. Presl. PhD Thesis, University of Granada, Granada, Spain. http://hdl.handle.net/10481/58616

Bennetzen, J.L., Park, M. 2018. Distinguishing friends, foes, and freeloaders in giant genomes. Curr. Opin. Genet. Dev., 49: 49-55.

Böhne, A., Brunet, F., Galiana-Arnoux, D., Schultheis, C., Volff, J.N. 2008. Transposable elements as drivers of genomic and biological diversity in vertebrates. Chromosome Res., 16: 203-215.

Bolger, A.M., Lohse, M., Usadel, B. 2014. Trimmomatic: a flexible trimmer for Illumina sequence data. Bioinformatics, 30: 2114-2120.

Bourgeois, Y., Boissinot, S. 2019. On the population dynamics of junk: a review on the population genomics of transposable elements. Genes, 10: 419.

Bourque, G., Burns, K.H., Gehring, M., Gorbunova, V., Seluanov, A., Hammell, M., Imbeault, M., Izsvák, Z., Levin, H.L., Macfarlan, T.S., Mager, D.L., Feschotte, C. 2018. Ten things you should know about transposable elements. Genome Biology, 19: 199.

Capy, P., Gasperi, G., Biémont, C., Bazin, C. 2000. Stress and transposable elements: coevolution or useful parasites. Heredity, 85: 101-106.

Chuong, E.B., Elde, N.C., Feschotte, C. 2017. Regulatory activities of transposable elements: From conflicts to benefits. Nature Review Genetics,18: 71-86.

Dyer, T.J., Pellicer, J., Savolainen, V., Leitch, I.J., Schneider, H. 2013. Genome size expansion and the relationship between nuclear DNA content and spore size in the Asplenium monanthes fern complex (Aspleniaceae). BMC Plant Biology, 13: 219. 

(Hymenophyllaceae: Pteridophyta), progress and future interests. Fern Gazette, 18: 53-58.

Faircloth, B.C. 2008. MSATCOMMANDER: Detection of microsatellite repeat arrays and automated, locus-specific primer design. Molecular Ecology Resources, 8: 92-94.

Feschotte, C. 2008. The contribution of transposable elements to the evolution of regulatory networks. Nat. Rev. Genet., 9: 397-405.

Fu, L., Niu, B., Zhu, Z., Wu, S., Li, W. 2012. CD-HIT: accelerated for clustering the nextgeneration sequencing data. Bioinformatics, 28: 3150-3152.

García-Guerreiro, M.P. 2012. What makes transposable elements move in the Drosophila genome? Heredity, 108: 461-468.

García-Guerreiro, M.P., Chávez-Sandoval, B.E., Balanyà, J., Serra, L., Fontdevila, A. 2008. Distribution of the transposable elements bilbo and gypsy in original and colonizing populations of Drosophila subobscura. BMC Evol. Biol. 8: 234.

García-Pérez, J.L., Widmann, T.J., Adams, I.R. 2016. The impact of transposable elements on mammalian development. Development, 143: 4101-4114.

Gregory, T.R. 2005. Genome size evolution in animals. In: Gregory T.R. (ed.) The Evolution of the Genome. Elsevier Academic Press, Burlington, USA, pp 3-87.

Hahn, C., Bachmann, L., Chevreux, B. 2013. Reconstructing mitochondrial genomes directly from genomic next-generation sequencing reads-a baiting and iterative mapping approach. Nucleic Acids Res., 41: e129.

Hřibová, E., Neumann, P., Matsumoto, T., Roux, N., Macas, J., Dolezel, J. 2010. Repetitive part of the banana (Musa acuminata) genome investigated by low-depth 454 sequencing. BMC Plant Biology, 10: 204.

Kalendar, R., Tanskanen, J., Immonen, S., Nevo, E., Schulman, A.H. 2000. Genome evolution of wild barley (Hordeum spontaneum) by BARE-1 retrotransposon dynamics in response to sharp microclimatic divergence. Proc. Nat. Acad. Sci. USA 97: 6603-6607.

Klekowski, E. 1972. Genetical features of ferns as contrasted with seed plants. Ann. Missouri Bot. Gard. 59: 138-151.

Klekowski, E.J., Baker, H.G. 1966. Evolutionary significance of polyploidy in the Pteridophyta. Science, 153: 305-307.

Li, F.W., et al. 2018. Fern genomes elucidate land plant evolution and cyanobacterial symbioses. Nature Plants, 4: 460-472.

Lockton, S., Ross-Ibarra, J., Gaut, B.S. 2008. Demography and weak selection drive patterns of transposable element diversity in natural populations of Arabidopsis lyrata. Proc. Nat. Acad. Sci. USA 105: 13965-13970. 
López-Flores, I., Garrido-Ramos, M.A. (2012) The repetitive DNA content of eukaryotic genomes. In: Garrido-Ramos, M.A. (ed.) Repetitive DNA. Genome Dynamics Volume 7. Karger, Basel, Switzerland, pp 126-152.

Lynch, M., Conery, J.S. 2003. The origins of genome complexity. Science, 302: 14011404.

Makałowski, W., Gotea, V., Pande, A., Makałowska, I. 2019. Transposable Elements: classification, identification, and their use as a tool for comparative genomics. In: Anisimova M (ed) Evolutionary Genomics: Statistical and Computational Methods, Methods in Molecular Biology Volume 1910. Springer Nature, Switzerland, pp 177-207.

Manton, I. 1950. Problems in cytology and evolution in the Pteridophyta. Cambridge University Press, Cambridge, 316 pp.

Manton, I., Lovis, J.D., Vida, G., Gibby, M. 1986. Cytology of the fern flora of Madeira. Bulletin of the British Museum (Natural History), Botany 15: 123-161.

Marchant, D.B., Sessa, E.B., Wolf, P.G., Heo, K., Barbazuk, W.B., Soltis, P.S., Soltis, D.E. 2019. The C-Fern (Ceratopteris richardii) genome: insights into plant genome evolution with the first partial homosporous fern genome assembly. Sci. Rep., 9: 18181.

Morgante, M., Brunner, S., Pea, G., Fengler, K., Zuccolo, A., Rafalski, A. 2005. Gene duplication and exon shuffling by helitron-like transposons generate intraspecies diversity in maize. Nature Genetics, 37: 997-1002.

Nakazato, T., Barker, M.S., Rieseberg, L.H., Gastony, G.J. 2008. Evolution of the nuclear genome of ferns and lycophytes. In: Ranker TA, Haufler CH (eds) Biology and Evolution of Ferns and Lycpohytes. Cambridge University Press, Cambridge, pp. 175-198.

Neumann, P., Novák, P., Hoštáková, N., Macas, J. 2019. Systematic survey of plant LTR retrotransposons elucidates phylogenetic relationships of their polyprotein domains and provides a reference for element classification. Mobile DNA, 10: 1.

Novák, P., Guignard, M.S., Neumann, P., Kelly, L.J., Mlinarec, J., Koblizkova, A., Dodsworth, S., Kovarik, A., Pellicer, J., Wang, W., Macas, J., Leitch, I.J., Leitch, A.R. 2020a. Repeatsequence turnover shifts fundamentally in species with large genomes. Nature Plants, 6: 13251329.

Novák, P., Neumann, P., Macas, J. 2010. Graph-based clustering and characterization of repetitive sequences in next-generation sequencing data. BMC Bioinformatics 11: 378.

Novák, P., Neumann, P., Macas, J. 2020b. Global analysis of repetitive DNA from unassembled sequence reads using RepeatExplorer2. Nature Protocols 15: 3745-3776. 

based web server for genome-wide characterization of eukaryotic repetitive elements from next generation sequence reads. Bioinformatics, 29: 792-793.

Novikov, A., Smyshlyaev, G., Novikova, O. 2012. Evolutionary history of LTR retrotransposon chromodomains in plants. International Journal of Plant Genomics, 2012: 874743.

497

Obermayer, R., Leitch, I.J., Hanson, L., Bennett, M.D. 2002. Nuclear DNA C-values in 30 species double the familial representation in pteridophytes. Ann. Bot. 90: 209-217.

Pryer, K.M., Schneider, H., Smith, A.R., Cranfill, R., Wolf, P.G., Hunt, J.S., Sipes, S.D. 2001. Horsetails and ferns are a monophyletic group and the closest living relatives to seed plants. Nature, 409: 618-622.

Ross-lbarra, J., Wright, S.I., Foxe, J.P., Kawabe, A., DeRose-Wilson, L., Gos, G., Charlesworth, D., Gaut, B.S. 2008. Patterns of polymorphism and demographic history in natural populations of Arabidopsis lyrata. PLoS ONE 2008: 3.

Ruiz-Ruano, F.J., López-León, M.D., Cabrero, J., Camacho, J.P.M. 2016. High-throughput analysis of the satellitome illuminates satellite DNA evolution. Sci. Rep. 6: 28333.

Ruiz-Ruano, F.J., Navarro-Domínguez, B., Camacho, J.P.M., Garrido-Ramos, M.A. 2019a. Characterization of the satellitome in lower vascular plants: the case of the endangered fern Vandenboschia speciosa. Ann. Bot. 123: 587-599.

Ruiz-Ruano, F.J., Navarro-Domínguez, B., Camacho, J.P.M., Garrido-Ramos, M.A. 2019b. Full plastome sequence of the fern Vandenboschia speciosa (Hymenophyllales): structural singularities and evolutionary insights. J. Plant. Res. 132: 3-17.

Rumsey, F.J., Vogel, J.C., Russell, S.J., Barrett, J.A., Gibby, M. 1999. Population genetics and conservation biology of the endangered fern Trichomanes speciosum (Hymenophyllaceae) in Scotland. Biol. J. Linn. Soc. 66: 333-344.

Schmieder, R., Edwards, R. 2011. Fast identification and removal of sequence contamination from genomic and metagenomic datasets. PLoS ONE 6: e17288.

Schneider, H., Schuettpelz, E., Pryer, K.M., Cranfill, R., Magallón, S., Lupia, R. 2004. Ferns diversified in the shadow of angiosperms. Nature, 428: 553-557.

Sessa, E.B., et al. 2014. Between Two Fern Genomes. Gigascience 2014: 3.

Sessa, E.B., Der, J.P. 2016. Evolutionary genomics of ferns and lycophytes. In: Advances in Botanical Research 78, Elsevier, pp. 215-254.

Slotkin, R.K., Martienssen, R. 2007. Transposable elements and the epigenetic regulation of the genome. Nat. Rev. Genet. 8: 272-285. 
Smit, A.F.A., Hubley, R., Green, P. 2015. RepeatMasker Open-4.0. 2013-2015. http://repeatmasker.org.

Smith, A.R., Pryer, K.M., Schuettpelz, E., Korall, P., Schneider, H., Wolf, P.G. 2006. A classification for extant ferns. Taxon 55: 705-731.

Thomas, J., Pritham, E.J. 2015. Helitrons, the eukaryotic rolling-circle transposable elements. Microbiology Spectrum 3: MDNA3-0049-2014.

Tollis, M., Boissinot, S. 2012. The evolutionary dynamics of transposable elements in eukaryote genomes. In: Garrido-Ramos, M.A. (ed.) Repetitive DNA. Genome Dynamics Volume 7. Karger, Basel, Switzerland, pp 68-97.

Vicient, C.M., Casacuberta, J.M. 2017. Impact of transposable elements on polyploid plant genomes. Ann. Bot. 120: 195-207.

Wagner, W.H., Wagner, F.S. 1980. Polyploidy in pteridophytes. In: Polyploidy. Springer, pp 199-214.

Weiss-Schneeweiss, H., Leitch, A.R., McCann, J., Jang, T.S., Macas, J. 2015. Employing next generation sequencing to explore the repeat landscape of the plant genome. In: Hörandl, E., Appelhans, M. (eds.) Next Generation Sequencing in Plant Systematics Regnum Vegetabile. Koeltz Scientific Books, Königstein, Germany, pp 155-179.

Wicker, T., Sabot, F., Hua-Van, A., Bennetzen, J.L., Capy, P., Chalhoub, B., Flavell, A., Leroy, P., Morgante, M., Panaud, O., Paux, E., SanMiguel, P., Schulman, A.H. 2007. A unified classification system for eukaryotic transposable elements. Nature Rev. Gen. 8: 973-982.

Wolf, P.G., Sessa, E.B., Marchant, D.B., Li, F.W., Rothfels, C.J., Sigel, E.M., Gitzendanner, M.A., Visger, C.J., Banks, J.A., Soltis, D.E., Soltis, P.S., Pryer, K.M., Der, J.P. 2015. An exploration into fern genome space. Genome Biol. Evol., 7: 2533-2544.

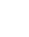

(1)

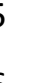


560 Figure legends

561

562 Figure 1. Curve profiles in the Repeat Landscapes (RL) of TEs for V. speciosa

563

564

565

566

567

568

569

570

571

572

573

574

575

(c) The Author(s) or their Institution(s) 


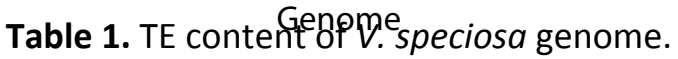

\begin{tabular}{|c|c|c|c|c|c|c|}
\hline \multicolumn{4}{|c|}{ TE taxonomy } & \multirow{2}{*}{ Abundance } & \multirow{2}{*}{$\begin{array}{l}\text { Abundance in } \\
\text { respect to all TEs }\end{array}$} & \multirow{2}{*}{$\begin{array}{l}\text { Abundance in } \\
\text { respect to } \\
\text { annotated TEs }\end{array}$} \\
\hline Class & Order & Superfamily & Family & & & \\
\hline \multirow[t]{24}{*}{ Class I } & LTR & & & $4,96 \%$ & $6,53 \%$ & $8,72 \%$ \\
\hline & & Ty1/Copia & & $9,90 \%$ & $13,02 \%$ & $17,40 \%$ \\
\hline & & & LTR/Copia-Ale & $4,27 \%$ & $5,62 \%$ & $7,50 \%$ \\
\hline & & & LTR/Copia-Tork & $2,43 \%$ & $3,20 \%$ & $4,27 \%$ \\
\hline & & & LTR/Copia-Gymcoll & $0,70 \%$ & $0,92 \%$ & $1,23 \%$ \\
\hline & & & LTR/Copia-GymcolV & $0,65 \%$ & $0,86 \%$ & $1,14 \%$ \\
\hline & & & LTR/Copia-Gymcolll & $0,58 \%$ & $0,76 \%$ & $1,02 \%$ \\
\hline & & & TOTAL COPIA & $18,53 \%$ & $24,38 \%$ & $32,57 \%$ \\
\hline & & Ty3/Gypsy & & $0,13 \%$ & $0,17 \%$ & $0,23 \%$ \\
\hline & & Non-chromovirus & LTR/Gypsy-OTA & $9,04 \%$ & $11,89 \%$ & $15,89 \%$ \\
\hline & & & LTR/Gypsy-OTA|Athila & $6,73 \%$ & $8,85 \%$ & $11,83 \%$ \\
\hline & & & LTR/Gypsy-OTA|Tat & $0,32 \%$ & $0,42 \%$ & $0,56 \%$ \\
\hline & & & LTR/Gypsy-OTA|Tat|Retand & $0,14 \%$ & $0,18 \%$ & $0,25 \%$ \\
\hline & & & TOTAL GYPSY NON-CHROMO & $16,23 \%$ & $21,35 \%$ & $28,52 \%$ \\
\hline & & Chromovirus & & $1,44 \%$ & $1,89 \%$ & $2,53 \%$ \\
\hline & & & LTR/Gypsy-CRM & $0,69 \%$ & $0,91 \%$ & $1,21 \%$ \\
\hline & & & LTR/Gypsy-Reina & $0,06 \%$ & $0,08 \%$ & $0,11 \%$ \\
\hline & & & LTR/Gypsy-Galadriel & $0,02 \%$ & $0,03 \%$ & $0,04 \%$ \\
\hline & & & TOTAL GYPSY CHROMO & $2,21 \%$ & $2,91 \%$ & $3,88 \%$ \\
\hline & & & TOTAL GYPSY & $18,57 \%$ & $24,43 \%$ & $32,64 \%$ \\
\hline & Total LTR & & & $42,06 \%$ & $55,33 \%$ & $73,92 \%$ \\
\hline & LINE & & & $8,71 \%$ & $11,46 \%$ & $15,31 \%$ \\
\hline & PLE & Penelope & & $0,54 \%$ & $0,71 \%$ & $0,95 \%$ \\
\hline & Caulimovirus & & & $0,03 \%$ & $0,04 \%$ & $0,05 \%$ \\
\hline Total Class I & & & & $51,34 \%$ & $67,54 \%$ & $90,23 \%$ \\
\hline \multicolumn{7}{|l|}{ Class II } \\
\hline \multirow[t]{4}{*}{ Subclass I } & EnSpm-CACTA & & & $3,46 \%$ & $4,55 \%$ & $6,08 \%$ \\
\hline & Sola1 & & & $2,00 \%$ & $2,63 \%$ & $3,51 \%$ \\
\hline & PIF-Harbinger & & & $0,01 \%$ & $0,01 \%$ & $0,02 \%$ \\
\hline & Total & & & $5,47 \%$ & $7,20 \%$ & $9,61 \%$ \\
\hline Subclass II & Helitron & & & $0,09 \%$ & $0,12 \%$ & $0,16 \%$ \\
\hline Total Class II & & & & $5,56 \%$ & $7,31 \%$ & $9,77 \%$ \\
\hline \multicolumn{2}{|c|}{ Total annotated elements } & & (c) The_Author(s)_or their_nstitution(s) & $56,90 \%$ & $74,86 \%$ & $100,00 \%$ \\
\hline Unknown & & & & $19,11 \%$ & $25,14 \%$ & \\
\hline TOTAL & & & & $76,01 \%$ & $100,00 \%$ & \\
\hline
\end{tabular}


Table 2. TE identification among non-annotated TEs of the genome of $V$. speciosa. Abundance: percentage in the genome of each non-annotated element but identified as a specific kind of TE by BLAST; Abundance in respect to Unknown elements: percentage of each identified TE in respect to the total of non annotated elements; Total identified elements: percentage of each TE in the genome of $V$. speciosa taken together both annotation and BLAST identification.

\begin{tabular}{|c|c|c|c|c|c|}
\hline \multicolumn{3}{|c|}{ TE taxonomy } & \multirow{2}{*}{ Abundance } & \multirow{2}{*}{$\begin{array}{l}\text { Abundance in } \\
\text { respect to } \\
\text { Unknown } \\
\text { elements }\end{array}$} & \multirow{2}{*}{$\begin{array}{c}\text { Total identified } \\
\text { elements (Annotation } \\
+ \text { Blast identification) }\end{array}$} \\
\hline Class & Order & Superfamily & & & \\
\hline \multirow[t]{7}{*}{ Class I } & LTR & & $0,38 \%$ & $1,99 \%$ & $5,34 \%$ \\
\hline & & Ty1/Copia* & $1,31 \%$ & $6,86 \%$ & $19,84 \%$ \\
\hline & & Ty3/Gypsy** & $4,38 \%$ & $22,92 \%$ & $22,95 \%$ \\
\hline & Total LTR & & $6,07 \%$ & $31,76 \%$ & $48,13 \%$ \\
\hline & LINE & & $0,87 \%$ & $4,55 \%$ & $9,58 \%$ \\
\hline & PLE & Penelope & $0,00 \%$ & $0,00 \%$ & $0,54 \%$ \\
\hline & Caulimovirus & & $0,00 \%$ & $0,00 \%$ & $0,03 \%$ \\
\hline Total Class & & & $6,94 \%$ & $36,32 \%$ & $58,28 \%$ \\
\hline \multicolumn{6}{|l|}{ Class II } \\
\hline \multirow[t]{4}{*}{ Subclass I } & EnSpm-CACTA & & $0,46 \%$ & $2,41 \%$ & $3,92 \%$ \\
\hline & Sola1 & & $1,04 \%$ & $5,44 \%$ & $3,04 \%$ \\
\hline & PIF-Harbinger & & $0,00 \%$ & $0,00 \%$ & $0,01 \%$ \\
\hline & Total & & $1,50 \%$ & $7,85 \%$ & $6,97 \%$ \\
\hline Subclass II & Helitron & & $0,00 \%$ & $0,00 \%$ & $0,09 \%$ \\
\hline Total Class & & & $1,50 \%$ & $7,85 \%$ & $7,06 \%$ \\
\hline Total anno & d elements & & $8,44 \%$ & $44,17 \%$ & $65,34 \%$ \\
\hline Unknown & & & $10,67 \%$ & $55,83 \%$ & $10,67 \%$ \\
\hline TOTAL & & & $19,11 \%$ & $100,00 \%$ & $76,01 \%$ \\
\hline
\end{tabular}

*(0.22\% Tork, $0.06 \%$ Ale, 0,04 Gymcoll, $0.03 \%$ Gymcolll and 0.06\% GymcollI)

**(4.24\% OTA |Athila, 1.86\% OTA, 0.15 OTA|Tat|Retand, 0.13 Chromovirus) 


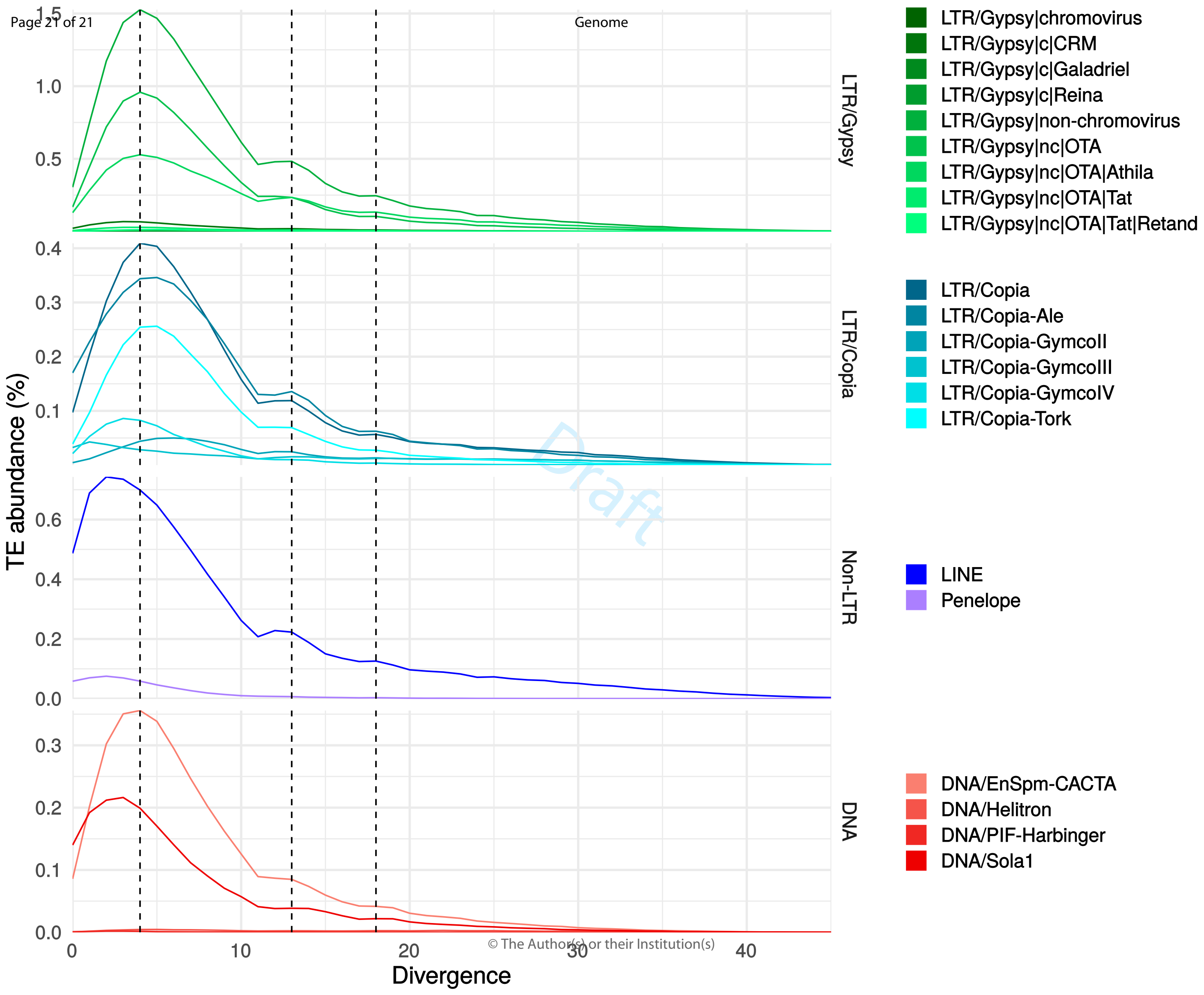

